\title{
Preface for the special issue on Frontier of Terahertz Technology
}

\author{
Toshitaka Idehara • Kiyomi Sakai • Masanori Hangyo • \\ Norihisa Hiromoto
}

Received: 30 November 2013 / Accepted: 3 December 2013 /

Published online: 12 December 2013

(C) Springer Science+Business Media New York 2013

The International Symposium on Frontier of Terahertz Technology (FTT 2012) was organized by the Committee on Terahertz Science and Industrial Development (in JSPS), the Terahertz Technology Forum of Japan and the University of Fukui. It was successfully held in November 2012 in Nara, Japan. The scope of the symposium is to discuss the current state of affairs across a broad range - from basic technologies to industrial applications - and clarify the prospect for the future. More than 200 participants were attending from over ten countries. They presented 34 papers in the plenary and oral sessions and 109 papers in the poster sessions. The subjects are extended to wide fields, such as the $\mathrm{THz}$ wave generation, $\mathrm{THz}$ components, THz spectroscopy, applications to astronomical and medical sensing and so on. Therefore, the contents of the symposium are really reflected the "Frontier of Terahertz Technology".

All of papers presented in FTT 2012 are interesting and impressive and indicating the future prospects of research and development on terahertz technologies. Among them, we selected 13 papers to organize this special issue. The first 6 papers are invited review papers, and other 7 papers are invited regular papers. We hope that these papers are attractive and useful for the whole readers of JIMT and trigger them the new ideas for development of terahertz science and technology in future.

November, 2013

Guest Editors of SI FTT 2012

T. Idehara $(\bowtie)$

University of Fukui, Fukui, Japan

e-mail: idehara@fir.u-fukui.ac.jp

K. Sakai

Communications Research Laboratory, Tokyo, Japan

M. Hangyo

Osaka University, Suita, Japan

N. Hiromoto

Shizuoka University, Shizuoka, Japan 